Check for updates

Cite this: RSC Adv., 2018, 8, 15310

\title{
A rapid and cost-effective metallization technique for 3C-SiC MEMS using direct wire bonding
}

\author{
Abu Riduan Md Foisal, (D) $\dagger^{\star a}$ Hoang-Phuong Phan, (D) $\dagger^{a}$ Toan Dinh, (D) a \\ Tuan-Khoa Nguyen, (D) a Nam-Trung Nguyen (D) and Dzung Viet Dao ab
}

This paper presents a simple, rapid and cost-effective wire bonding technique for single crystalline silicon carbide (3C-SiC) MEMS devices. Utilizing direct ultrasonic wedge-wedge bonding, we have demonstrated for the first time the direct bonding of aluminum wires onto $\mathrm{SiC}$ films for the characterization of electronic devices without the requirement for any metal deposition and etching process. The bonded joints between the $\mathrm{Al}$ wires and the $\mathrm{SiC}$ surfaces showed a relatively strong adhesion force up to approximately 12.6-14.5 $\mathrm{mN}$ and excellent ohmic contact. The bonded wire can withstand high temperatures above $420 \mathrm{~K}$, while maintaining a notable ohmic contact. As a proof of concept, a 3C-SiC strain sensor was demonstrated, where the sensing element was developed based on the piezoresistive effect in $\mathrm{SiC}$ and the electrical contact was formed by the proposed direct-bonding technique. The SiC strain sensor possesses high sensitivity to the applied mechanical strains, as well as exceptional repeatability. The work reported here indicates the potential of an extremely simple direct wire bonding method for SiC for MEMS and microelectronic applications.

Received 24th January 2018 Accepted 12th April 2018

DOI: $10.1039 / \mathrm{c} 8 \mathrm{ra00734a}$

rsc.li/rsc-advances mechanism to remove the surface oxide and contaminations from the film..$^{\mathbf{9} 13}$ Generally, for silicon based devices, the wire bonding is performed to connect metal wires to metal electrodes that are pre-deposited and patterned on the surface of silicon. A metal pad is required as $\mathrm{Si}$ has a relatively high oxidation rate, and it forms a native oxide layer, hindering the contact between the metal wire and the Si layer. However, a number of recent studies successfully demonstrated that direct wire bonding is feasible in wide band gap materials, such as GaN, owing to its excellent chemical inertness, which prevents the oxidation process occurring on the surface at room temperature. ${ }^{8}$ The capability of direct wire bonding offers notable advantages, and as such, it allows the characterization of materials right after the growth process, and also eliminates the metal depositing and etching process to significantly simplify the whole fabrication process for MEMS devices. ${ }^{\mathbf{1 4}}$ Furthermore, direct wire bonding also enables the formation of electrical contacts for MEMS suspended structures (such as membranes, cantilever beams, and doubly-clamped bridges) formed by wet-etching, where the deposition and etching of metals on these released structures are impractical.

Employing the superior chemical inertness of SiC, this work demonstrates a fast, simple, and low-cost metallization technique using direct $\mathrm{Al}$ wire bonding onto a SiC surface. The proposed technique provides easy and efficient access to the characterization of electrical properties (for instance, Hall effect measurement) in epitaxial SiC films right after the CVD deposition process, and is applicable to the development of $\mathrm{SiC}$ based MEMS.

\footnotetext{
${ }^{a}$ Queensland Micro-Nanotechnology Centre, Griffith University, Queensland, Australia. E-mail:ar.mdfoisal@griffithuni.edu.au

${ }^{b}$ School of Engineering, Griffith University, Queensland, Australia

$\dagger$ These authors contributed equally to this work.
} 


\section{Device fabrication and experimental setup}

A $280 \mathrm{~nm}$ thick p-type 3C-SiC material was grown on a Si (100) substrate (which has a carrier concentration of $5 \times 10^{14} \mathrm{~cm}^{-3}$ and resistivity of $1 \Omega \mathrm{cm}$ ) using low pressure chemical vapor deposition at $1000^{\circ} \mathrm{C} .{ }^{15,16}$ Trimethylaluminium (TMAl) was used as a source of p-type dopants in the in situ doping process. From the Hall measurement, the carrier concentration of the grown film was found to be $5 \times 10^{18} \mathrm{~cm}^{-3}$ and the measured resistivity was $0.14 \Omega \mathrm{cm} .{ }^{17}$ Subsequently, $3 \mathrm{C}-\mathrm{SiC}$ resistors were fabricated using inductively coupled plasma etching with an etch rate of $100 \mathrm{~nm} \mathrm{~min}{ }^{-1}$ in $\mathrm{HCl}$ and $\mathrm{O}_{2}$ plasma. ${ }^{18}$ The wafer was then diced into strips with dimensions of $80 \mathrm{~mm} \times 8 \mathrm{~mm} \times$ $0.625 \mathrm{~mm}$ to induce uniaxial stress in the [110] direction employing the bending method. Prior to wire bonding, the diced beam was cleaned with acetone and isopropanol and then dried out with nitrogen gas. Direct wire bonding was then applied to the SiC surface to connect the SiC resistors to the external copper-PCB electrodes using a wedge-wedge wire bonder (747630E-79, West Bond Inc.). The ultrasonic power was set at $350 \mathrm{~mW}$ for a duration of $30 \mathrm{~ms}$ to perform the $\mathrm{Al}$ wire to $\mathrm{SiC}$ bonding. To measure the pulling strengths of the bonded $\mathrm{Al}$ wires on the 3C-SiC surface, a manual pulling test (destructive mode) was performed with an electronic mass balance (FX3000i, A\&D Company Ltd.). $I-V$ characteristics of the $3 \mathrm{C}-\mathrm{SiC}$ sensor at high temperatures were measured using a semiconductor device analyzer (U2722A, Agilent Tech.) and a dehydrating oven (TD-500F, Thermoline Scientific).

\section{Results and discussion}

A Bruker ${ }^{\mathrm{TM}}$ D8 advance X-ray diffraction (XRD) system was used to provide $\operatorname{CuK} \alpha$ emission in the full $2 \theta-\theta$ range of the XRD measurement. The acquisition angles of the $2 \theta-\theta$ scan were in the range of $30^{\circ}$ to $90^{\circ}$ with an increment of $0.005^{\circ}$ per step. The XRD measurement shows diffraction peaks at $35.6^{\circ}$ and $90^{\circ}$, corresponding to the $3 \mathrm{C}-\mathrm{SiC}(200)$ and $3 \mathrm{C}-\mathrm{SiC}$ (400) orientations, as shown in Fig. 1. Additionally, besides these two peaks, only a peak at $69.1^{\circ}$, corresponding to Si (400), was observed. This result indicates that the SiC film was epitaxially grown on the (100) Si substrate. Furthermore, the Selected Area Electron Diffraction (SAED) image (inset of Fig. 1) also confirms that the grown SiC film is single crystalline.

Fig. 2(a) shows an SEM image of the SiC resistors, where the first bond was formed on $\mathrm{SiC}$ and the second bond was formed on an external copper-PCB pad. The inset of Fig. 2(a) illustrates an SEM image of the tail of the wire bond formed on the SiC film with a surface area of approximately $50 \mu \mathrm{m} \times 50 \mu \mathrm{m}$. Fig. 2(b) shows the wire bonding where both first and second bonds were formed on the SiC surface, which can be utilized to interconnect different devices on the same chip. Fig. 2(c) shows the experimental setup to measure the bond strength between the $3 \mathrm{C}-\mathrm{SiC}$ surface and the Al wires. The pulling force was measured by mounting a bonded SiC/Si chip onto an electronic mass balance (both first and second bonds were connected on the $3 \mathrm{C}-\mathrm{SiC}$

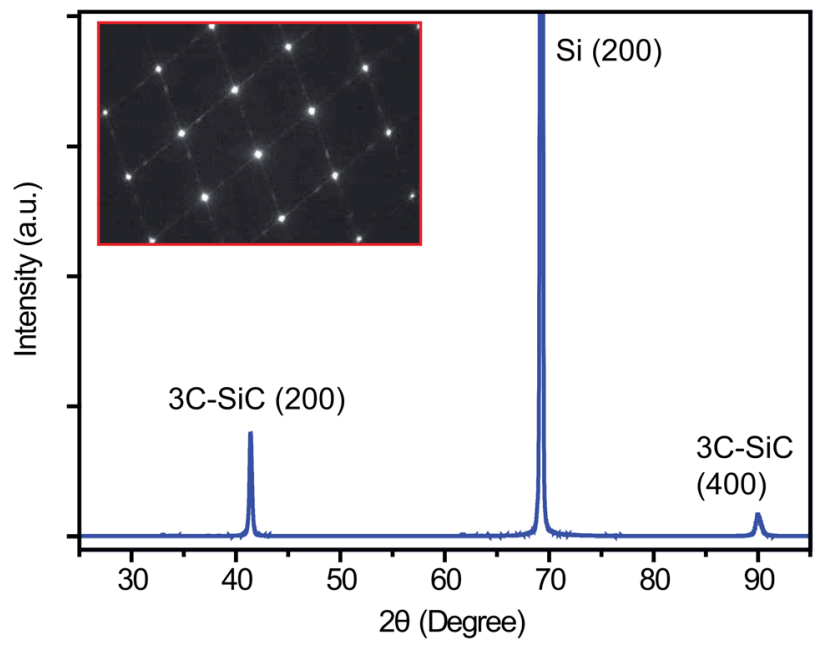

Fig. 1 (a) X-ray diffraction pattern of the grown $3 \mathrm{C}-\mathrm{SiC}$ film on a $\mathrm{Si}$ (100) substrate. The inset shows the SAED image.

surface as shown in Fig. 2(b)). Subsequently, a micro-positioner with a hook-tip was employed to pull the center of the wire with each moving step being set at $3 \mu \mathrm{m}$. The applied pulling-force was monitored using an electronic balance; the bonding strength was found when the wire started to detach from the SiC surface. Accordingly, the bond strength ranged from 12.6-14.5 $\mathrm{mN}$.

Similarly, for comparison, the bond strength of the directly bonded $\mathrm{Al}$ wire on the deposited $\mathrm{Al}$ contact of $\mathrm{SiC}$ was also measured and it was observed that the strength was in the range of $31.2-38.6 \mathrm{mN}$, which is $59-62 \%$ higher than that between the
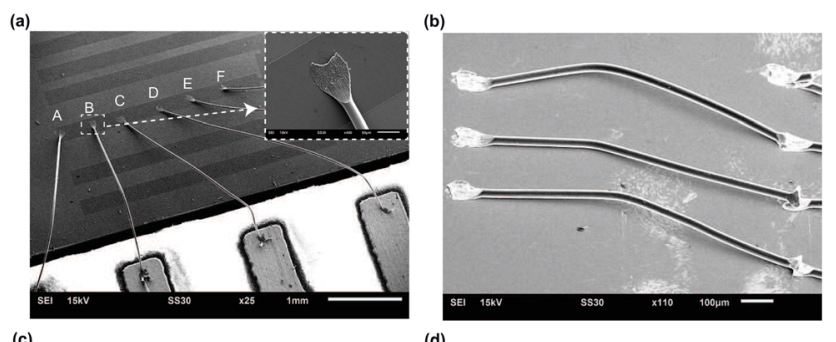

(c)

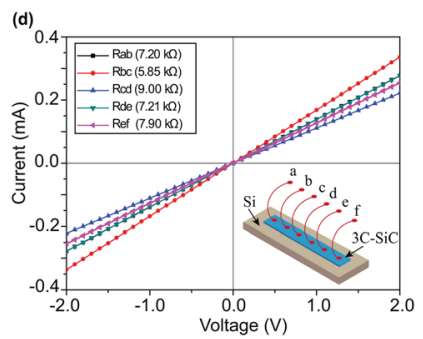

Fig. 2 (a) SEM image of the directly bonded Al wire between the 3CSiC surface and a metal pad (the inset shows the zoomed-in view of the contact area formed by direct Al wire bonding), (b) demonstrates the possibility of bonding both first and second bonds on the same SiC surface, (c) schematic of the experimental setup to measure the bond strength (destructive mode), and (d) $I-V$ characteristic curves of different SiC resistors (each resistor has different spacing between the bonded areas). 
directly bonded $\mathrm{Al}$ wire and the 3C-SiC surface. However, the bond strength between the direct $\mathrm{Al}$ wire bonding and the $\mathrm{SiC}$ surface is comparable to that of the standard minimum bond pull limit $(14.7 \mathrm{mN})$ of $\mathrm{Al}$ wires that are $25 \mu \mathrm{m}$ in diameter. ${ }^{19}$

The electrical properties of the bonded wire shown in Fig. 2(d) were then investigated using the Agilent ${ }^{\mathrm{TM}}$ U2722A analyzer. The linear behavior of the current-voltage characteristics represents an excellent ohmic contact between the directly bonded $\mathrm{Al}$ wires and the 3C-SiC surface, as shown in Fig. 2(d). Furthermore, it is also evident that under a constantly applied voltage, the measured current was found to be inversely proportional to the distance between the bonded pads. Subsequently, the contact resistance between the $\mathrm{Al}$ wire and the $3 \mathrm{C}-$ SiC film was also measured using a TLM (Transmission Line Measurement) technique and the contact resistivity was found to be approximately $3.32 \times 10^{-3} \Omega \mathrm{cm}^{2}$ (Fig. 3).

We also compared the resistance of the SiC resistors fabricated using the bonding method to that using the standard one with the metal pad formed by aluminium deposition and etching. The top inset in Fig. 4 shows a SiC resistor with metal electrodes, where an Al layer with a thickness of $300 \mathrm{~nm}$ was sputtered using Surrey Nano Systems- $\gamma$. From the $I-V$ curve (red line in Fig. 4), the resistance of the $\mathrm{SiC}$ with $\mathrm{Al}$ electrodes was found to be $1210 \mathrm{k} \Omega$. Subsequently, the deposited $\mathrm{Al}$ electrodes were completely removed using TMAH, and the direct wire bonding was performed on $\mathrm{SiC}$, as shown in the bottom inset of Fig. 4. The resistance of the SiC resistor formed using direct wire bonding (solid blue line) was found to be almost the same as that of the $\mathrm{SiC}$ resistors with the $\mathrm{Al}$ electrode. This indicates the small contact resistance of direct bonding, and also implies the possibility of using this novel method for SiC MEMS applications without the requirement for metal electrodes on SiC.

In addition, to test the feasibility of using direct wire bonding on 3C-SiC devices under high temperature environments, the 3C-SiC film was transferred from the Si substrate onto a glass substrate using an anodic bonding process, ${ }^{\mathbf{1 , 2 0}}$ to

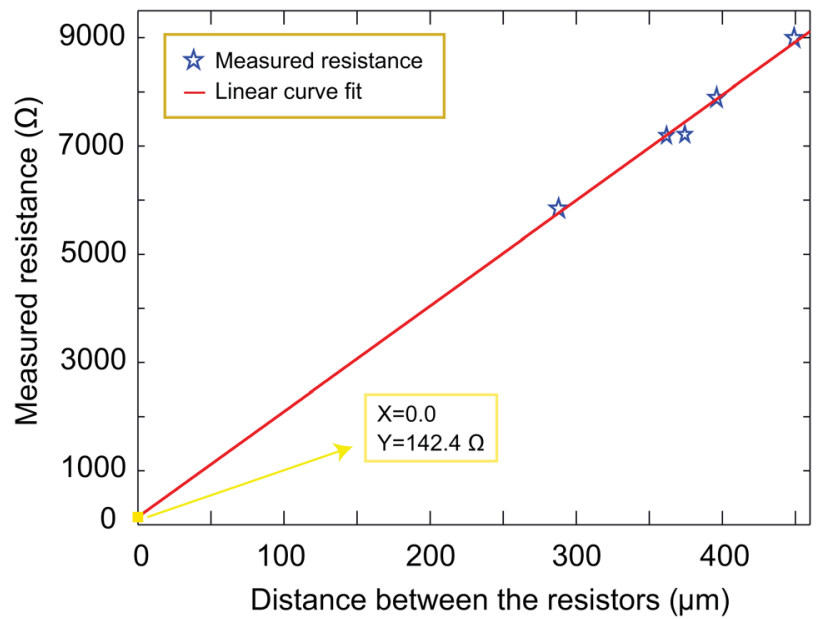

Fig. 3 Measurement of contact resistance utilizing the Transmission Line Measurement technique.

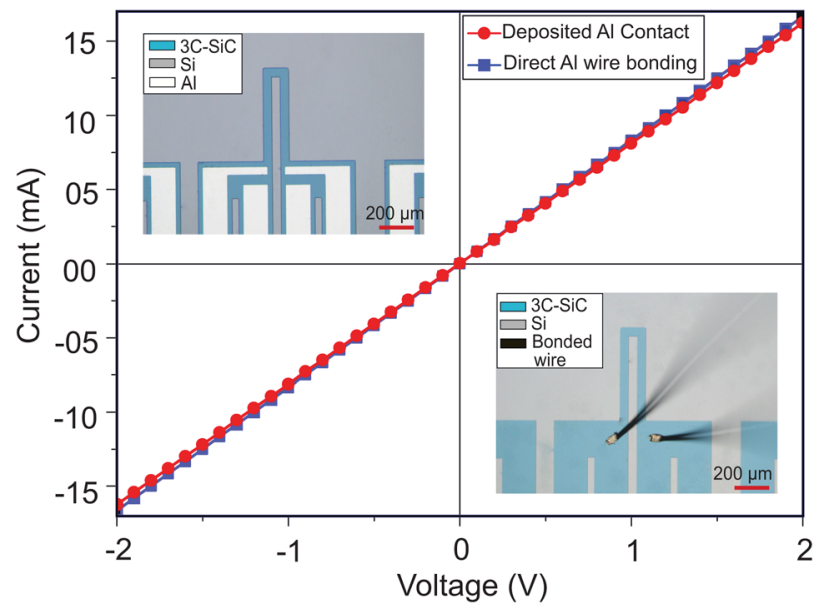

Fig. $4 \quad I-V$ characteristics of a $3 C-S i C$ resistor with a deposited $\mathrm{Al}$ contact and with direct Al wire bonding (after removing the deposited contact via wet etching). The optical micrographs of both configurations are shown in the top and bottom insets, respectively.

prevent the leakage through the $\mathrm{SiC} / \mathrm{Si}$ junction at high temperatures. $^{21,22}$ The electrical contacts were then prepared by direct wire bonding to the transferred 3C-SiC film. As observed in the $I-V$ characteristics (Fig. 5), the current through the $3 \mathrm{C}-$ $\mathrm{SiC}$ resistor increased as the temperatures were raised, and the contact between the $\mathrm{Al}$ wire and the $\mathrm{SiC}$ resistor retained its linear behavior at high temperatures, which is a desired property for applications at elevated temperatures. Furthermore, after cooling down to room temperature, the device showed the same $I-V$ characteristics as under the initial room temperature conditions, demonstrating the repeatable and stable behavior of the device at high temperatures.

To demonstrate the application of the direct wire bonding method, a SiC strain sensor was developed utilizing the piezoresistive effect. The bottom inset of Fig. 5 shows a SiC on Si

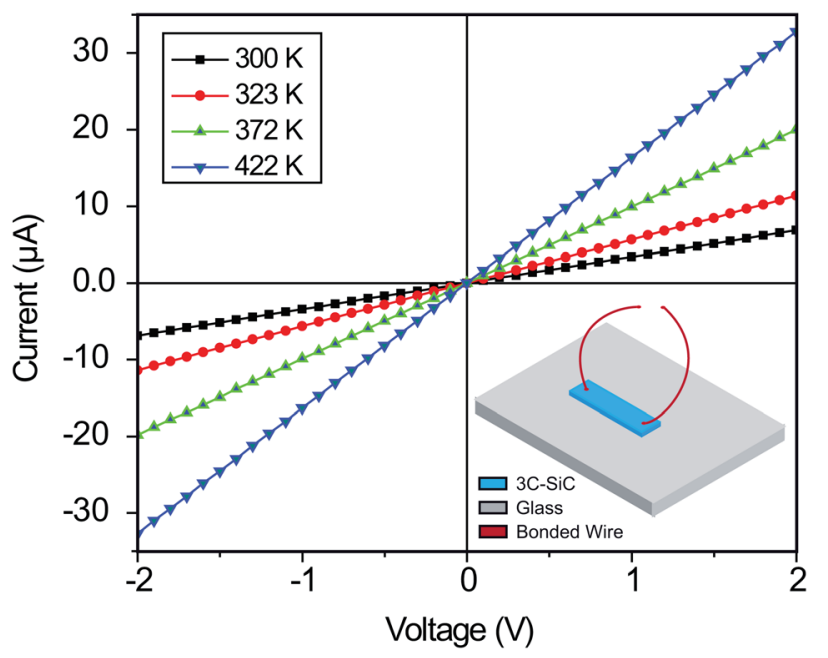

Fig. 5 Current-voltage response of a $3 \mathrm{C}-\mathrm{SiC}$ resistor on a glass substrate at different temperatures. The inset shows a schematic of the $3 \mathrm{C}-\mathrm{SiC} /$ glass resistor. 


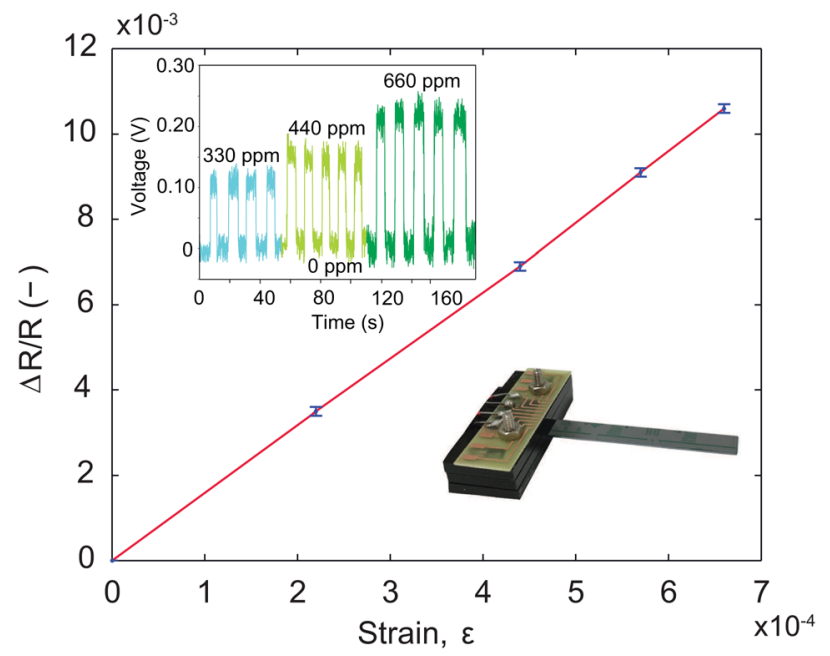

Fig. 6 Relationship between the relative resistance change of the SiC resistor at different strains. The top inset represents the real-time response of the device under various strain levels and the fabricated $\mathrm{SiC} / \mathrm{Si}$ beam for the bending experiment is shown in the bottom inset.

cantilever, where the SiC strain sensors were patterned into Ushape structures, as illustrated in the inset of Fig. 4. Direct wire bonding was employed to make contact with the copper PCB placed on the clamp of the SiC/Si cantilever. The piezoresistive effect of the fabricated $3 \mathrm{C}-\mathrm{SiC}$ resistor was measured using the bending beam method, where the uniaxial stress was induced in the [110] direction. ${ }^{23,24}$ It was observed that the relative resistance change increased linearly with increasing strain. This is a desirable property for p-type 3C-SiC, as the uniaxial stress was applied in the longitudinal direction, which means that the direction of the current flowing through the resistor and that of the applied stress were the same. Furthermore, the responses showed excellent repeatability at constant strain levels, as shown in the top inset of Fig. 6.

\section{Conclusions}

In conclusion, this study successfully demonstrated the feasibility of direct wire bonding for 3C-SiC MEMS devices, such as strain sensors for high temperature applications. It is observed experimentally that the bonded wires were physically and electrically stable under stress and high temperature conditions. Direct wire bonding also provides quick and easy access to the electrical properties of a 3C-SiC film just after CVD deposition. In addition, the metallization of SiC MEMS devices with direct $\mathrm{Al}$ wire bonding is more cost-effective and time-efficient compared to that of the conventional metallization process, which requires expensive and complicated fabrication processes. Therefore, the study presented here promises an efficient, cost-effective, and mechanically stable wiring technique for 3C-SiC based MEMS devices using direct wire bonding.

\section{Conflicts of interest}

There are no conflicts to declare.

\section{Acknowledgements}

This work was performed in part at the Queensland node of the Australian National Fabrication Facility, a company established under the National Collaborative Research Infrastructure Strategy to provide nano- and microfabrication facilities for Australia's researchers. This work has been partially supported by Griffith University's New Researcher Grants and Australian Research Council grants LP150100153 and LP160101553.

\section{References}

1 H.-P. Phan, H. H. Cheng, T. Dinh, B. Wood, T.-K. Nguyen, F. Mu, H. Kamble, R. Vadivelu, G. Walker, L. Hold, A. Iacopi, B. Haylock, D. V. Dao, M. Lobino, T. Suga and N.-T. Nguyen, ACS Appl. Mater. Interfaces, 2017, 9, 2736527371.

2 S. Liu, H. Liu, Z. Huang, M. Fang, Y. G. Liu and X. Wu, RSC Adv., 2016, 6, 24267-24272.

3 D. V. Dao, H.-P. Phan, A. Qamar and T. Dinh, RSC Adv., 2016, 6, 21302-21307.

4 A. Qamar, H.-P. Phan, D. V. Dao, P. Tanner, T. Dinh, L. Wang and S. Dimitrijev, IEEE Electron Device Lett., 2015, 36, 708710.

5 A. R. M. Foisal, A. Qamar, H.-P. Phan, T. Dinh, T.-K. Nguyen, P. Tanner, E. W. Streed and D. V. Dao, ACS Appl. Mater. Interfaces, 2017, 9, 39921-39925.

6 A. R. M. Foisal, H.-P. Phan, T. Kozeki, T. Dinh, T.-K. Nguyen, A. Qamar, M. Lobino, T. Namazu and D. V. Dao, RSC Adv., 2016, 6, 87124-87127.

7 H.-P. Phan, A. Qamar, D. V. Dao, T. Dinh, L. Wang, J. Han, P. Tanner, S. Dimitrijev and N.-T. Nguyen, RSC Adv., 2015, 5, 56377-56381.

8 H. So and D. G. Senesky, Appl. Surf. Sci., 2016, 387, 280-284.

9 Y. Takahashi and M. Inoue, J. Electron. Packag., 2002, 124, 27-36.

10 J. Y. Lee, Y. C. Chen and M. S. Lin, Method of wire bonding over active area of a semiconductor circuit, U.S. Pat., 9,142,527, 2015.

$11 \mathrm{~N}$. Zommer, Solderless die attach to a direct bonded aluminum substrate, U.S. Pat., 8,716,864, 2014.

12 B. Gehman, IEEE Trans. Compon., Hybrids, Manuf. Technol., 1980, 3, 375-383.

13 G. Harman and J. Albers, IEEE Trans. Parts, Hybrids, Packag., 1977, 13, 406-412.

14 H. So and D. G. Senesky, J. Phys. D: Appl. Phys., 2016, 49, 285109.

15 L. Wang, S. Dimitrijev, J. Han, P. Tanner, A. Iacopi and L. Hold, J. Cryst. Growth, 2011, 329, 67-70.

16 S. A. Kukushkin and A. V. Osipov, J. Phys. D: Appl. Phys., 2014, 47, 313001.

17 H.-P. Phan, D. V. Dao, P. Tanner, L. Wang, N.-T. Nguyen, Y. Zhu and S. Dimitrijev, Appl. Phys. Lett., 2014, 104, 111905.

18 A. Qamar, H.-P. Phan, J. Han, P. Tanner, T. Dinh, L. Wang, S. Dimitrijev and D. V. Dao, J. Mater. Chem. C, 2015, 3, 8804-8809. 
19 Department of Defense, MIL-STD-883E Test Method Standard Microcircuits, Method 2011.7, 1996.

20 C. Tudryn, S. Schweizer, R. Hopkins, L. Hobbs and A. J. Garratt-Reed, J. Electrochem. Soc., 2005, 152, E131-E134.

21 M. Placidi, A. Pérez-Tomás, M. Zielinski, P. Godignon, J. Millán, X. Jordà, N. Mestres and G. Abadal, J. Micromech. Microeng., 2010, 20, 115007.
22 C.-H. Wu, C. A. Zorman and M. Mehregany, IEEE Sens. J., 2006, 6, 316-324.

23 H.-P. Phan, T. Dinh, T. Kozeki, T.-K. Nguyen, A. Qamar, T. Namazu, N.-T. Nguyen and D. V. Dao, Appl. Phys. Lett., 2016, 109, 123502.

24 H.-P. Phan, T.-K. Nguyen, T. Dinh, G. Ina, A. R. Kermany, A. Qamar, J. Han, T. Namazu, R. Maeda, D. V. Dao and N.-T. Nguyen, Appl. Phys. Lett., 2017, 110, 141906. 